\title{
EDITORIAL
}

\section{Administrative database research}

\author{
John R. W. Kestle, MD \\ Department of Neurosurgery, University of Utah, Salt Lake City, Utah
}

$\mathrm{I}$ $\mathrm{N}$ this issue, Parker et al. ${ }^{4}$ report their analysis of data from a large multi-institutional source called the Premier Perspective Database. This database includes billing and discharge information from inpatient stays and outpatient visits at more than 600 US hospitals. More than 10,000 adults and 1770 children undergoing shunt insertion are included, and antibiotic-impregnated catheters (AICs) were used in about $10 \%$ (8.9\% of adults, $12.9 \%$ of children). The authors found reduced infection rates with (vs without) AICs (adults 2.2\% vs 3.6\%; children $2.5 \%$ vs $7.1 \%$ ). These results are similar to those reported for other nonrandomized studies, but the use of a large administrative database is relatively new to this field. It is therefore worthwhile to discuss some of the methodology issues that may arise.

Data sets derived from billing and coding data have become readily available and often provide large numbers of patients and events. The large sample size in these studies results in a high power to detect treatment effects and allows multivariate adjustment for risk factors that is often not possible in smaller clinical studies. On the other hand, this method of data acquisition introduces new sources of potential error that have not been discussed much in the neurosurgical literature. Some of these limitations are described by the authors, but a few others are worth mentioning:

1. Data source. Usually the basis for administrative data sets is billing information. If a clinical event is not necessary for billing purposes, it may be missing or inaccurate. The more relevant the information is for the billing and coding process, the more likely it is to be included accurately. ${ }^{1}$ Coders use the medical record to derive information and generate the billing codes. They are therefore dependent on the accuracy and completeness of the medical record, which is subject to all of the uncertainties and inaccuracies that we are familiar with from retrospective clinical research.
In addition, when the data are obtained from insurance claims, information will be captured only for patients and events that are covered at that time. Patients and events will be missed if their insurance coverage lapses. ${ }^{3}$ A description of the extent and consistency of coverage is therefore important in these studies.

2. Coding issues. Typically coders assign one primary and one or more secondary diagnoses. The choice of primary and secondary diagnosis can vary. For example, a child with a brain tumor and hydrocephalus could have either condition chosen as the primary diagnosis. The primary diagnosis may be brain tumor even when the child is admitted for a shunt revision. This choice can be made differently depending on the circumstances and can result in events being missed or not counted. ${ }^{5}$

3. Linkages. It appears that the Premier Perspective Database obtains information from multiple sources. In that case, the process of linking the data sources becomes very important. Detailed algorithms are often required to make sure that events over time, from multiple sources and possibly from multiple sites, are correctly linked to the same patient. Validation of the linkage process is important to ensure data accuracy. ${ }^{3}$

4. Data validation. Administrative databases are not designed to answer research questions. Ideally, their data sets should be validated against other data sources. For example a subset of the data could be compared to a disease-specific patient registry that was designed for research on the condition. In addition, the set of criteria that are used to define the sample should be validated. This is to ensure that the set of codes used to extract information from the database accurately pulls what the researchers want. In this case the codes should have identified all patients undergoing shunt surgery, the use of AICs, and the outcome (infected or not).

5. Confounders. The large sample size allows for power- 
ful statistical modeling, but any statistical model is only able to adjust for the data fields that are available. ${ }^{3}$ In this case, some of the factors that are associated with shunt infection are not available for assessment; examples of such factors include CSF leak, age (prematurity), and comorbid conditions.

6. Definition of infection. This database includes information from hundreds of hospitals. A common code is used for infection, but the criteria for assigning that code are not described. In other words, the definition of infection may vary from one site to another and is potentially subject to observer bias.

Are these issues a concern in the study by Parker et al.? It is difficult to tell-some of the information necessary for making this determination is not available.

The use of AICs is gaining a lot of attention. The evidence published to date appears to me to be suggestive but not definitive. A randomized trial comparing standard catheters, AICs, and silver-impregnated catheters is well under way in Europe. ${ }^{2}$ It has nonindustry, peer-reviewed funding, adequate sample size and power, and excellent oversight and trial management by experienced clinical trialists. I will await the results before deciding whether or not to change my practice.

http://thejns.org/doi/abs/10.3171/2014.4.JNS14689

\section{References}

1. Gavrielov-Yusim N, Friger M: Use of administrative medical databases in population-based research. J Epidemiol Community Health 68:283-287, 2014

2. Mallucci C, Jenkinson M, Hartley J, Gamble C, Hickey H, Dwan K, et al: The British antibiotic and silver impregnated catheters for ventriculoperitoneal shunts multi-centre randomised controlled trial (the BASICs trial). Childs Nerv Syst 28:1602, 2012 (Abstract)

3. Motheral B, Brooks J, Clark MA, Crown WH, Davey P, Hutchins D, et al: A checklist for retrospective database studies-report of the ISPOR Task Force on Retrospective Databases. Value Health 6:90-97, 2003

4. Parker SL, McGirt MJ, Murphy JA, Megerian JT, Stout M, Engelhart L: Comparative effectiveness of antibiotic-impregnated shunt catheters in the treatment of adult and pediatric hydrocephalus: analysis of 12,589 consecutive cases from 287 US hospital systems. J Neurosurg [epub ahead of print November 21, 2014. DOI: 10.3171/2014.10.JNS13395]

5. Vaughan Sarrazin MS, Rosenthal GE: Finding pure and simple truths with administrative data. JAMA 307:1433-1434 2012

\section{Response \\ Scott L. Parker, MD, ${ }^{1}$ and Matthew J. McGirt, MD ${ }^{2}$}

'Department of Neurosurgery, Vanderbilt University Medical Center, Nashville, Tennessee; and ${ }^{2}$ Carolina Neurosurgery \& Spine Associates, Charlotte, North Carolina

We would like to thank Dr. Kestle for his thoughtful editorial regarding our article. He eloquently identifies and discusses the limitations inherent in studies that utilize information obtained from administrative databases. We agree that such data should be viewed in light of these limitations, as detailed in the Discussion section of our paper.

The results of our current study are not meant to singularly provide definitive evidence on the effectiveness of AICs, but rather to add to the growing body of results that have suggested that use of AICs is associated with a reduction in shunt infection. The findings of the current study are consistent with those of previous systematic reviews and meta-analyses of the literature, which found antibiotic-impregnated shunt catheters to be associated with a reduced incidence of CSF infection., ${ }^{1,2}$ Even with their limitations, administrative databases provide a means of assessing real-world care in large, representative patient populations and can be particularly useful for a research question involving a relatively rare event in which large patient populations are required in order to appreciate a significant change based on the interventions of interest (i.e., the effect on infection incidence following the introduction of AICs). It is in these situations that we believe non-Level 1 evidence studies can still provide valuable insight to clinicians, especially when viewed in aggregate as a body of evidence on a particular research subject. Nevertheless, randomized controlled trials remain the gold standard for best-quality medical evidence, and we too look forward to the results of the ongoing trial assessing this important question.

\section{References}

1. Parker SL, Anderson WN, Lilienfeld S, Megerian JT, McGirt MJ: Cerebrospinal shunt infection in patients receiving antibiotic-impregnated versus standard shunts. J Neurosurg Pediatr 8:259-265, 2011

2. Thomas R, Lee S, Patole S, Rao S: Antibiotic-impregnated catheters for the prevention of CSF shunt infections: a systematic review and meta-analysis. Br J Neurosurg 26:175184,2012 